\title{
EchoGéo
}

30 | 2014

Varia

\section{Approche spatiale d'un centre hospitalier, le CHU pédiatrique de Ouagadougou (Burkina Faso)}

\section{Aude Nikiema}

\section{(2) OpenEdition}

1 Journals

\section{Electronic version}

URL: https://journals.openedition.org/echogeo/14046

DOI: $10.4000 /$ echogeo. 14046

ISSN: 1963-1197

\section{Publisher}

Pôle de recherche pour l'organisation et la diffusion de l'information géographique (CNRS UMR 8586)

\section{Electronic reference}

Aude Nikiema, "Approche spatiale d'un centre hospitalier, le CHU pédiatrique de Ouagadougou (Burkina Faso)", EchoGéo [Online], 30 | 2014, Online since 11 December 2014, connection on 31 July 2021. URL: http://journals.openedition.org/echogeo/14046 ; DOI: https://doi.org/10.4000/echogeo. 14046

This text was automatically generated on 31 July 2021.

EchoGéo est mis à disposition selon les termes de la licence Creative Commons Attribution - Pas d'Utilisation Commerciale - Pas de Modification 4.0 International (CC BY-NC-ND) 


\title{
Approche spatiale d'un centre hospitalier, le CHU pédiatrique de Ouagadougou (Burkina Faso)
}

\author{
Aude Nikiema
}

Ce travail a été réalisé dans le cadre de l'étude intitulée "L'enfant dans les services de pédiatrie en Afrique de l'Ouest » (UMI 3189 Environnement, santé, société). Nous remercions le coordinateur principal, le Professeur Y. Jaffré, et la coordinatrice pays, M.-T. Arcens Somé au Burkina Faso, pour nous avoir permis de porter un regard géographique sur ce lieu. Les recherches ont été menées à l'hôpital pédiatrique de Ouagadougou grâce à la collaboration active du Professeur Yé Diarra et du Dr A. Kaboré, toutes deux pédiatres dans cet établissement et membres de l'équipe. Nous remercions également E. Bonnet pour ses conseils relatifs à l'approche géographique.

\section{Introduction}

1 «La santé est un état de complet bien-être physique, mental et social, et ne consiste pas seulement en une absence de maladie ou d'infirmité » (OMS, 1946). Cette définition suppose de centrer les politiques de santé « sur la personne dans son environnement " et d'associer des déterminants sociaux aux déterminants de santé (Fleuret, 2003).

2 Or, les politiques de santé mises en œuvre en Afrique au cours des décennies ne répondent qu'en partie à cette définition. Malgré les efforts entrepris, les hôpitaux conservent une mauvaise réputation. Celle-ci témoigne «de l'ampleur des dysfonctionnements que connaissent les formations sanitaires, et de la dégradation des relations entre soignants et soignés » (Jaffré et al., 2005, p. 11). Ces dysfonctionnements s'inscrivent dans un cadre plus large de conversion du monde médical observé ailleurs et qui conduit l'hôpital vers une gestion technocratique qui participe de « la spirale de défiance qui abîme les relations entre les soignants, certains administratifs et les patients » (Mas et al., 2011, p. 11). 
3 Ces dysfonctionnements n'empêchent pas l'hôpital moderne d'être fréquenté. En Afrique, son accès est souvent jugé inéquitable en raison du rôle significatif joué par les relations sociales et le niveau économique des patients (de Roodenbeck, 2003). L'hôpital apparaît comme un univers hostile et non rassurant pour tout usager dépourvu de connaissances et d'argent (Ebang Ondo, 2012). Structure faitière de la hiérarchie sanitaire, dans les pays africains, le centre hospitalier universitaire est une étape dans le processus de la quête de guérison dans un milieu marqué par un pluralisme médical. Les composantes du large éventail de recours aux soins disponibles sont utilisées au gré des maux vécus et interprétés par les individus. Les itinéraires thérapeutiques sont donc hétérogènes et complexes. Cet état de fait peut expliquer que parfois les plus pauvres sautent l'étape du dispensaire pour aller directement à l'hôpital (Nikiema et al, 2011) alors que d'autres ne le rejoindront qu'après plusieurs échecs thérapeutiques (Djouda Feudjio, 2010).

4 La place particulière tenue par l'hôpital dans l'itinéraire de soins des malades se retrouve également dans l'organisation spatiale des soins. Elle est marquée par l'apparition du district sanitaire, unité géographique fonctionnelle de décentralisation de l'organisation sanitaire. Ce district est destiné à « établir une communication avec la population et une participation des communautés» (Chenge Mukalenge, 2013). L'introduction de ce découpage s'inscrit dans un mouvement actuel de territorialisation de la politique de santé. Toutefois, le Centre hospitalier universitaire (CHU), en tant qu'établissement de référence disposant d'un plateau technique de pointe, se démarque par son aire de responsabilité qui échappe aux districts et couvre un espace bien plus vaste. Mais il fonctionne également comme hôpital de proximité, au sens propre du terme, ouvert sur son environnement.

5 L'urbanisation accroît les besoins d'hôpitaux (Bridgeman cité par George, 1966). Il est devenu un service urbain central parce que la croissance des villes le rattrape (CantalDupart, 2012). L'histoire de l'hôpital atteste du lien avec la politique urbaine et de sa place dans les grandes options d'urbanisme (Clémençon, 2002). Il devient donc un lieu où les aménageurs et architectes tentent de "concilier les exigences d'une gestion moderne avec les impératifs d'un authentique service public» (Manga Zambo, n.d., p. 4). Les autorités administratives et sanitaires ont pour volonté de faire de l'hôpital un objet urbain (Labasse, 1980). À l'échelle de la ville, de nombreux travaux montrent un pôle de référence, de formation universitaire, principal pourvoyeur d'emplois (Fleuret, 2012). À l'échelle infrahospitalière, l'observation reste plus rare (Granier, 2002 , ;Vaguet et al, 2012). Pourtant, celle-ci laisse deviner des enjeux importants en termes d'utilisation et de qualité des soins. Elle permet de repérer la structuration du lieu et d'expliquer les espaces de pratiques.

6 Ces constats témoignent d'un changement d'échelle d'observation très prégnant dans les politiques de soins des pays du nord et qui semble échapper au sud. À une échelle fine, le lieu hospitalier est marqué par l'évolution des formes architecturales destinée à répondre à la fonction hygiéniste des premiers établissements. Mais, aujourd'hui, les patients et leurs proches portent à l'égard de l'hôpital, un jugement bien plus attaché à des faits anodins qu'aux succès des traitements (Herranz, Thomas, 2009). L' " humanisation" des soins à l'hôpital fait place à des expériences dont les plus répandues se traduisent par une dimension matérielle du bâti (architecture, agencement, création d'espaces conviviaux) (Briand, Pary, 2011). La dimension relationnelle avec les soignants, mais aussi avec les lieux est progressivement incluse. 
Selon la charte européenne de l'enfant hospitalisé, cet établissement doit fournir aux enfants «un environnement correspondant à leurs besoins physiques, affectifs et éducatifs » (UNAF, 2007).

7 Au Sud, les lieux de soins manquent d'humanité (Luisier, 2011). Dans ces pays, la maladie peut être considérée comme « une affaire éminemment sociale » qui engage un certain type de représentation (Dozon, 2014). On peut alors s'interroger sur les liens pouvant exister entre la compréhension de la géographie des lieux de l'hôpital par les familles et le bien-être des patients.

La création des hôpitaux en Afrique est marquée de l'empreinte des modèles architecturaux occidentaux. Au Burkina Faso, dans la capitale, le Centre hospitalier universitaire Yalgado (1962) et le Centre hospitalier universitaire pédiatrique Charles de Gaulle (1997) présentent des modèles certes différents par la taille des bâtiments, mais offrent une physionomie proche du patrimoine hospitalier européen. Toutefois, si la forme est respectée, le fonctionnement les hôpitaux burkinabè semble figé dans un modèle qui évolue pourtant au Nord. Cela se traduit par une amélioration des conditions d'accueil et d'hospitalisation qui s'organise autour de trois types d'acteurs : le personnel hospitalier, les patients et les visiteurs (Burton, n.d.). Or, dans les hôpitaux, et notamment en Afrique, le patient est perçu comme un cas médical, mais pas dans un contexte social et humain (Briand, Pary, 2011). Les prises affectives des lieux, qui participent à leur intégration dans le territoire urbain et déterminent leur pratique par les populations (Audas, 2012), sont ainsi occultées.

9 Nous faisons donc l'hypothèse que le modèle organisationnel de l'hôpital burkinabè, vient en contradiction avec les modes de fonctionnement communautaire d'une majorité de la population et conduit à une incompréhension du fonctionnement des lieux qui peut nuire au bien-être des patients et leurs accompagnants.

10 Afin de vérifier cette hypothèse, les travaux théoriques de deux auteurs ont servi de guide pour décrire les lieux de l'hôpital burkinabè. Ainsi, l'approche de Gesler (1992) sur le concept de "therapeutic landscapes" a été retenue. Il décrit le paysage hospitalier comme faisant partie du processus de guérison du patient (Gesler et al, 2004). «La valeur thérapeutique d'un lieu de soins serait liée à la combinaison de ses dimensions physique, sociale et symbolique» (Gesler et al., 2004; Petit, 2010). Des projections identitaires conduisent à des attentes spécifiques sur l'organisation des lieux et interrogent également sur l'image renvoyée par ce lieu (Larceneux, 2011). La démarche s'enrichit également du concept de «lieux synaptiques", développé par Granier (2002), dont la fonction est d'engendrer des effets de seuil et « répond à une volonté de gestion des individus dans l'espace hospitalier formant un territoire ultranormé ». L'approche géographique doit ainsi contribuer à la compréhension de l'organisation spatiale de l'hôpital burkinabè par les populations et à la définition de son image jugée, plus largement en Afrique, « inhospitalière » (Jaffré, Olivier de Sardan, 2005).

11 Dans un premier temps, nous explorerons le territoire du Centre hospitalier universitaire pédiatrique de Ouagadougou pour le décrire dans une perspective organisationnelle. Les déplacements seront ensuite observés au sein de l'établissement pour compléter notre approche du territoire hospitalier. Enfin, nous esquisserons un modèle d'organisation fondé sur des seuils, des frontières, des espaces d'exclusion, etc. qui contribuent à modeler le paysage thérapeutique de l'hôpital implanté depuis plusieurs décennies au Burkina Faso. 


\section{Méthodologie} 4 commerçants, 14 sont non scolarisés et 3 ont un niveau primaire. Parmi les résidents urbains, les activités économiques sont très diversifiées : 4 couturières, 7 commerçants, enseignant, journaliste, accoucheuse auxiliaire, etc., 6 sans emploi et 4 élèves/ étudiants. Le niveau scolaire de ces parents est le suivant : 9 sont non scolarisés, 13 ont atteint le primaire et 11 le niveau secondaire.

afin de comprendre comment les familles des patients se déplaçaient sur le territoire hospitalier. Elle s'articulait avec une enquête socio-anthropologique dans laquelle des plans étaient associés, destinés à mettre en évidence les lieux pratiqués et les itinéraires de déplacements.

Compte tenu de l'âge très variable des patients selon les unités de soins et du souhait de disposer d'informations sur la perception de l'organisation externe des bâtiments, seuls les accompagnants des enfants ont été interrogés. Ce sont les patients de trois unités de soins dénommées au sein du CHUP «nourrissons ", "grands enfants » (plus de deux 
ans) et «maladies infectieuses », en fonction des activités de soins spécifiques menées, qui ont fait l'objet de nos enquêtes.

\section{Le Centre hospitalier universitaire pédiatrique, un territoire à explorer}

19 Le choix architectural de l'hôpital pédiatrique de Ouagadougou, fondé sur un modèle pavillonnaire au détriment de l'hôpital bloc, a sans doute autant pour objectif de respecter les règles d'hygiénisme que de l'intégrer à la ville.

20 L'hôpital pédiatrique de Ouagadougou regroupe les cinq grands secteurs qui composent l'établissement depuis le milieu du XXe siècle : service médico technique, hébergement, service logistique, service administratif, service technique du bâtiment. Il est construit de sorte que le visiteur le découvre en trois temps.

21 Les bâtiments de première ligne, situés sur le même alignement que la barrière de contrôle des entrées, correspondent au domaine administratif et financier, dont la direction générale (Illustration 1). Payer, gérer les soins (admission), gérer l'hôpital (administration), aider les patients (aide sociale) sont la vocation de ces bâtiments. Bien que situés à l'entrée, ils ne deviennent visibles aux visiteurs qu'à la sortie, en raison de leurs fonctions et de leur intervention tardive au cours de l'itinéraire hospitalier. Ils semblent contrôler les mouvements tant vers l'intérieur que vers l'extérieur au terme du circuit d'hospitalisation. Cette organisation pourrait être comparée à celle d'un commerce répondant physiquement au profil économique que la réforme hospitalière souhaite attribuer aux hôpitaux sur le territoire (Balique, 2004). Granier (2002) reprenant les propos de Frémont (1981) montre que les bâtiments abritant l'administration sont généralement associés aux lieux de pouvoir, "un pouvoir qui s'affiche et se matérialise le plus souvent par une expression symbolique ». À l'hôpital pédiatrique, cette symbolique se retrouve dans la position des bâtiments. Situés de part et d'autre de l'entrée, ils s'offrent comme des sentinelles autorisant la sortie des patients au terme d'une procédure financière : le règlement des frais hospitaliers. Cette position renforce l'idée que l'organisation interne de l'établissement adhère aux principes économiques dévolus à l'hôpital. 
Illustration 1- Disposition des bâtiments et couloirs de circulation au CHU pédiatrique

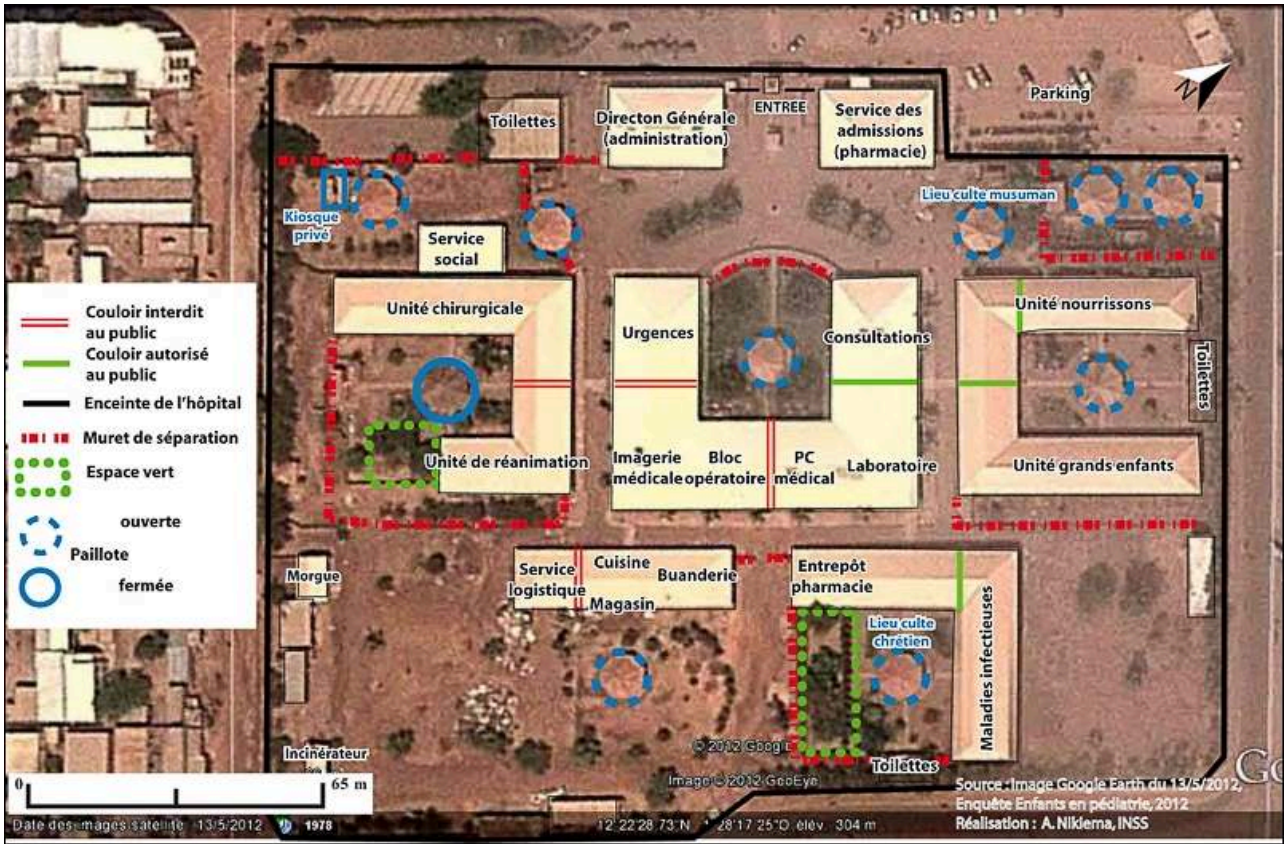

Une fois la barrière de contrôle passée (première ligne), le patient se dirige vers les

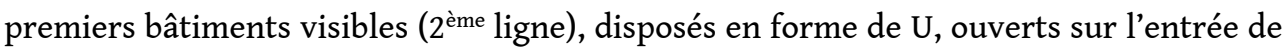
l'hôpital. Cette progression semble obligatoire. L'agencement du site est organisé pour limiter la déviation du regard, comme la présence de murets de part et d'autre du U (illustration 1). La partie gauche du U accueille les services de consultation et la partie droite, le service des urgences. Ils sont reliés par le bloc opératoire limité d'un côté par le laboratoire et de l'autre l'imagerie médicale. Sur le même alignement, cette forme géométrique est prolongée par des bâtiments de part et d'autre, disposés également en U, mais ouverts sur les murs d'enceinte. Ainsi, vers le nord, les bâtiments sont occupés par les unités destinées aux grands enfants et aux nourrissons, et vers le sud, se trouvent les unités de réanimation et chirurgicale. Ces constructions se situent au cœur de la cour et le cheminement naturel des visiteurs les conduit vers eux. Par ailleurs, la proximité relative des services vise à limiter les déplacements du personnel imposés par l'éparpillement des constructions et les larges couloirs de circulation.

Enfin, en troisième ligne, au fond de la cour de l'hôpital se trouvent les bâtiments destinés à accueillir les enfants touchés par les maladies infectieuses, l'entrepôt pharmaceutique et les services de logistique. La position relativement excentrée de cette unité thérapeutique correspond à l'image que revêt encore l'hôpital, celui d'un lieu source de risque.

L'urbanité de l'hôpital est mise en évidence par la recherche d'un accès facilité, par la volonté de diriger les flux, organiser le repos des visiteurs et faciliter les rencontres. L'établissement est structuré de façon à prendre toutes les étapes de l'itinéraire thérapeutique hospitalier en charge. Pharmacie, laboratoire, radiologie doivent éviter aux patients et leur famille de recourir aux services de la ville. Cette prise en charge se retrouve au travers des aspects financiers puisqu'un forfait couvre les frais d'examen quel que soit le nombre réalisé.

Malgré le choix d'un modèle architectural pavillonnaire, l'organisation interne de l'espace hospitalier et les matériaux de construction constituent une forme de 
distanciation entre l'individu et les services offerts. Ainsi, l'ensemble des enquêtés résidant en milieu rural parle des différences entre l'organisation de l'espace hospitalier et celle du village. Certains évoquent le modèle de construction avec des bâtiments plus grands et disposés de façon complexe, sans distinction possible, ce qui entraîne une confusion et une perte de repères :

«J'ai du mal à retrouver les services, je demande à chaque fois qu'on me les indique », «la cour est grande, c'est très difficile pour quelqu'un qui n'a pas fait l'école », « les bâtiments sont complexes, quand on sort on ne sait plus revenir » (H.O. et A.B., 2012).

L'homogénéité apparente des bâtiments ne permet pas aux individus de se déplacer, l'esthétique a primé sur l'information en conservant une unité de couleur. Les personnes interrogées évoquent l'analphabétisme d'un grand nombre d'utilisateurs, l'existence d'une signalétique ne constituerait donc pas un moyen efficace pour tous de différencier les lieux et il n'existe aucun moyen d'orientation alternatif. Enfin, l'impression d'égarement pourrait être résumée dans la citation d'un enquêté résidant dans la capitale :

"L'architecture est faite de façon fermée, ce qui empêche les gens de se retrouver »

(A. S., 2012).

\section{La simplicité des itinéraires de circulation, l'enfermement vécu dans un espace clos?}

Au cours des enquêtes, les lieux visités et les axes empruntés pour y accéder ont été dessinés par un accompagnant sur un plan. Ces itinéraires, souvent réalisés après plusieurs jours d'hospitalisation, ne permettent pas de retranscrire précisément les hésitations, les questionnements lors de l'arrivée des patients. Mais ils mettent en évidence les contraintes vécues (illustration 2).

L'absence de repères physiques, d'indications écrites peu explicites et incompréhensibles pour les non-alphabétisés, explique que les nouveaux arrivants, soient souvent perdus dès le passage $d u$ portail d'entrée. Ils sont alors systématiquement dirigés par les vigiles, qui n'ont qu'un rôle d'orientation, mais pas de fonction médicale, vers le service des urgences même si la situation ne demande qu'une simple consultation. D'autre part, la simplicité des itinéraires révèle la crainte de se perdre (illustration 2). Les déplacements sont limités au strict minimum selon des axes toujours identiques (chaque couleur représente un nouveau déplacement), imposés par des couloirs de circulation dont certains sont interdits au public. Les flux se concentrent toujours dans la même partie de l'espace hospitalier mettant en évidence, par négatif, les espaces dont l'accès est limité. Ces déplacements sont liés aux soins, comme l'achat des médicaments ou la réalisation d'examens. Concernant ces derniers, ils sont généralement accompagnés d'un agent de liaison qui assure, pour le malade, l'acheminement des examens dans certains cas, ou le mène jusqu'au lieu de prélèvement. 
Illustration 2 - Schéma de circulation d'un patient de l'unité « grands enfants »

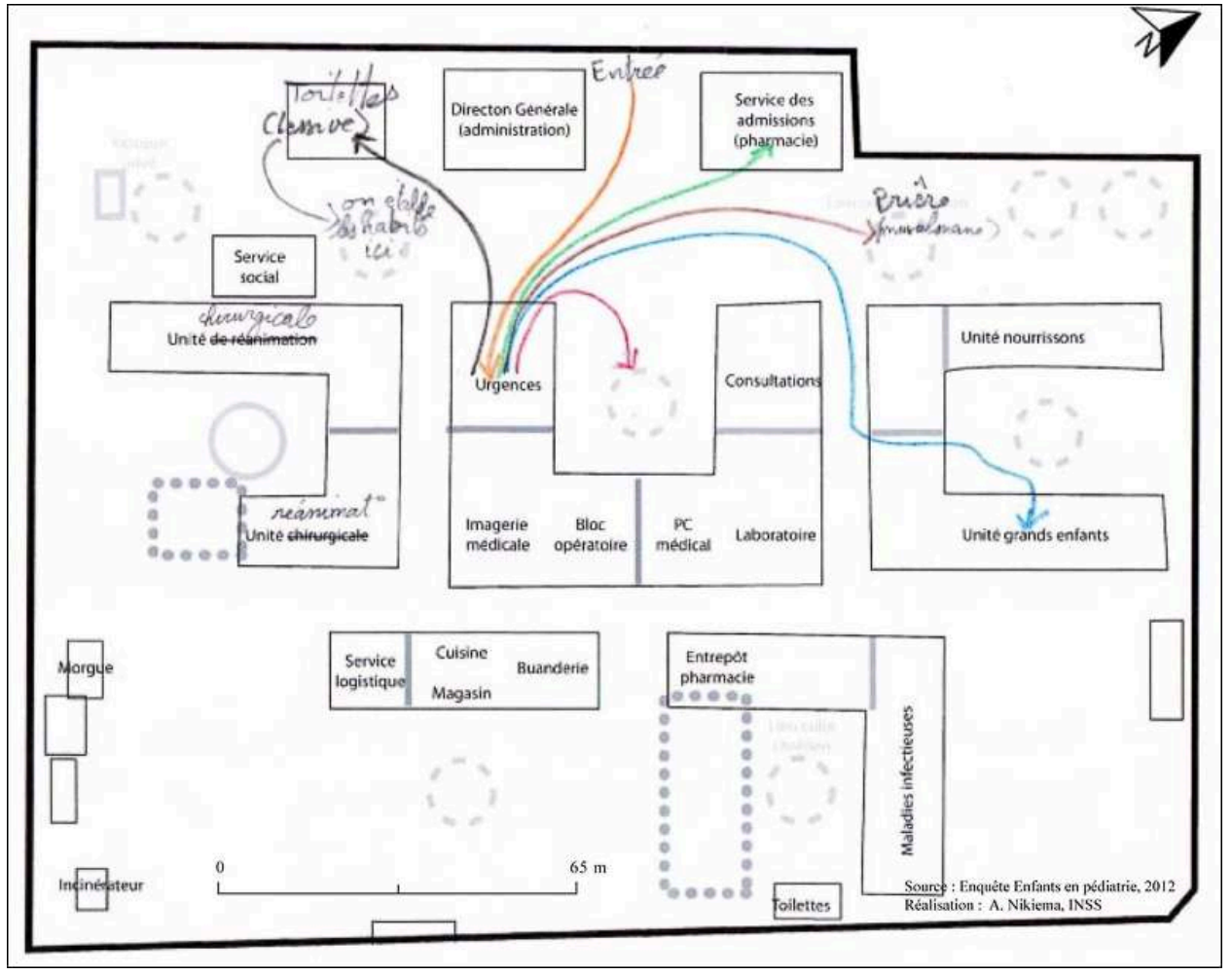

Les contraintes semblent intériorisées (Durand, Le Floch, 2006). En effet, le système de circulation dans l'hôpital n'est pas évoqué comme un problème par les enquêtés. À la question s'intéressant aux endroits non accessibles dans l'hôpital, les axes de circulation interdits ne sont jamais évoqués. Les personnes ayant répondu à cette question la transcrivent en terme médical. Elles associent le laboratoire, la chirurgie ou l'unité de réanimation à des lieux "d'expérience", de contamination, ou qui nécessitent « une aération » du malade. La présence du vigile dans les couloirs pour interdire le passage n'est pas perçue comme une contrainte. Le vigile est davantage perçu comme une personne susceptible d'informer, de diriger vers les lieux difficiles à retrouver. Cette intériorisation des contraintes est telle chez certains enquêtés qu'ils déclarent :

«L'hôpital est un lieu public on peut aller là où on veut, rien n'est interdit ».

On s'interroge alors sur l'image et le positionnement de l'hôpital aux yeux des familles des malades.

31 Ces itinéraires matérialisent l'enfermement dans ses formes les plus abstraites résultant d'une sorte de va-et-vient entre l'intériorité et l'extériorité (Di Méo, 2008). L'intériorité pourrait être assimilée aux règles de l'hôpital imposée par une architecture (murets, couloirs) et la surveillance (vigiles) régulant les flux et l'extériorité matérialisée par une tendance à s'approprier l'espace public pour le valoriser privativement (Shadyc-Gril, 2002), ce que les autorités de l'hôpital veulent éviter.

Les lieux fréquentés par le public se résument donc au strict minimum. Notons que les paillotes, présentes auprès de chaque unité de soins (matérialisées par des cercles bleus illustration 1), constituent le lieu, non lié aux soins, systématiquement fréquenté par les accompagnants. Par leur architecture, elles s'inscrivent davantage dans l'espace 
quotidiennement vécu par les familles. Ce sont de petits hangars recouverts d'une toiture dont le sous-bassement est formé par un mur et la partie haute par de simples piliers permettant de laisser passer l'air. En ville et surtout au village, les familles passent plus de temps à l'extérieur des bâtiments. L'aménagement des chambres avec de grandes fenêtres ne change pas le sentiment de coupure avec l'extérieur. La propreté des lieux, la présence de végétation basse, invitent par ailleurs le visiteur à se limiter à ces lieux aménagés pour leur détente et à ne pas s'installer dans des espaces considérés non appropriés par la Direction de l'hôpital.

Pour favoriser le bien-être des patients et leur famille, la difficulté est de passer d'un espace conçu comme simple support de l'action humaine à un espace conçu comme une construction de la société (Loschak, 2007). Plusieurs éléments ponctuant l'espace hospitalier ne plaident pas en faveur d'un tel changement. La difficulté réside sans doute dans les origines très hétérogènes des patients de l'hôpital pédiatrique de Ouagadougou. Son caractère unique sur le territoire burkinabè et sa vocation en font un établissement fréquenté tant par les populations urbaines que rurales.

Les itinéraires matérialisent, également, la perception des lieux et l'image que renvoie leur organisation, éléments participant à la guérison des patients. Ils contribuent à la construction d'un paysage thérapeutique qui peut être, selon les catégories socioéconomiques ou l'origine géographique des individus, en discordance avec les représentations de l'espace de vie fondé sur la communauté.

\section{Vers un modèle de territoire hospitalier?}

Aujourd'hui, la conception des lieux est de plus en plus réfléchie à des fins de bien-être, facteur participant à la guérison (Andrade et al, 2012; Curtis et al, 2007). En lien avec cette appréhension de la santé, le concept de paysage thérapeutique a fait l'objet de nombreuses publications de la part des géographes de la santé au Royaume-Uni où l'architecture hospitalière connaît un regain d'intérêt (Petit, 2010). Des travaux destinés à révéler l'importance de la composition des lieux dans la guérison ont été menés dans les hôpitaux psychiatriques (Curtis et al, 2007) ou dans les structures de prises en charge des maladies de neuro dégénérescence (Vaguet et al, 2012).

L'organisation de l'espace hospitalier pédiatrique à Ouagadougou s'appuie sur plusieurs structures : une succession de lieux accessibles à tous, organisés de sorte à plus ou moins limiter les mouvements, et de lieux non accessibles reliés par des lignes (les couloirs) chargées d'orienter les déplacements. Ces lieux sont à la fois issus de choix architecturaux et de la relation de pouvoir existant entre les personnes exerçant au sein de la structure et les patients. Ce paysage hospitalier est ponctué de constructions à la charge symbolique et qui participent à l'impact thérapeutique des aménagements sur les patients.

\section{De la frontière, rupture des déplacements...}

La médicalisation de l'architecture (Borasi, 2012) de l'hôpital montre que son organisation est pensée dans le but de limiter les contagions et les transferts vers la ville. Àl'intérieur du site, la décision est également prise de séparer les services et donc les bâtiments de façon à limiter les risques nosocomiaux. L'hôpital change d'échelle et se divise en unités (Fermand, 2002). 
Dans l'hôpital burkinabè, ces choix se matérialisent par plusieurs éléments. Le plus remarquable est le mur d'enceinte qui marque une frontière tant du point de vue externe, qu'interne. Ce mur reflète l'architecture de l'habitat urbain local, de plainpied. Sa fonction protectrice marquée en centre-ville s'estompe en périphérie pour ne marquer que le territoire, comme au village (Déverin, 1999). À l'hôpital, le mur symbolise la limite avec l'extérieur, la ville. Les déplacements n'y sont plus libres mais soumis à l'organisation interne. Celle-ci impose «virtuellement» des axes de circulation aux personnes par la disposition des bâtiments et leur accessibilité. L'accès à tous les lieux n'est pas possible, au risque de diffuser des germes pathogènes.

Malgré l'absence d'une signalétique adaptée, tout le monde n'a pas la liberté de prendre le chemin qu'il souhaite. Les lieux sont porteurs de frontières invisibles mais perçues par les individus présents dans l'enceinte de l'hôpital qui les conduira à limiter leurs déplacements essentiellement en lien avec leurs soins spécifiques. Les autres unités seront naturellement évitées par la présence de murets qui imposent une frontière psychologique mais pourtant franchissable puisqu'il existe un point d'entrée ouvert en permanence. Ces limites confèrent «sens et fonction" (Moullé, 2010) aux espaces concernés.

D'autres lieux sont porteurs de frontières visibles, équivalentes à des points de rupture structurelle. Elles sont matérialisées par la présence de vigiles qui interdit tout passage non autorisé. L'accès au territoire hospitalier devient discontinu.

41 La frontière matérialise donc la séparation entre deux espaces, l'un accessible en permanence, l'autre soumis à conditions. Elle est conçue comme un système de contrôle des flux (Moullé, 2010). Dans le cas du CHU Pédiatrique, elle institue une distinction par l'appartenance matérielle et symbolique à une entité territoriale: l'unité d'hospitalisation.

\section{...à la frontière synonyme d'enfermement}

L'enjeu réside donc dans l'adaptation des populations à l'organisation de l'espace et aux choix architecturaux, dont la vocation première est d'assurer une aération des lieux et la circulation des hommes. Il existe une contradiction entre un modèle de vie occidental individualiste, dont sont issues les formes hospitalières burkinabè, et un modèle de vie africain tourné vers la communauté, comme au Burkina Faso. La description de ces lieux vise à mettre en évidence « la distinction sociale et politique de tout espace et de sa territorialisation, en conclusion, un espace clos assimilable à un espace d'enfermement» (Di Méo, 2008). Cet enfermement, qui implique la suppression d'une sortie, n'est interrompu que par les dysfonctionnements de l'hôpital pédiatrique qui obligent le contact avec l'extérieur afin d'acheter médicaments et nourriture. On peut alors avancer, comme pour les gated communities, qu'il s'agit d'un espace fermé mais pas d'enfermement. Cependant, Di Méo met en avant l'idée « que vivre dans un tel habitat [gated communities] reflète une sorte d'enfermement social et psychologique». Une image dont relèverait le CHUP. L'enfermement perçu en ce lieu est identifié par la présence de vigiles à la sortie de l'hôpital. Sans entraver la sortie, ceux-ci marquent symboliquement le passage du lieu hospitalier à la ville.

43 À l'échelle des unités de soins et leur accès, l'enfermement peut être défini comme la " volonté, sociale et politique, visant à renforcer une position matérielle, s'inscrivant dans une stratégie; expression d'un pouvoir cherchant l'appui et le renfort d'une 
spatialisation ». Ce pouvoir se matérialise par le « jeu de l'interdit et de l'autorisation d'accès à l'espace protégé et contrôlé par la maîtrise de la porte »(Di Méo, 2008, p. 8). La présence des vigiles illustre le fonctionnement des unités chirurgie, réanimation, PC médical etc. et le pouvoir médical qui impose une forme d'isolement des patients mais également du personnel soignant. La structure de la paillote dans cette partie de l'hôpital est représentative de l'isolement et de la limitation des contacts avec l'extérieur que le service médical souhaite imposer. En effet, à l'exception d'une porte, elle est complètement fermée et occupée en permanence par les familles. L'architecture des bâtiments participe ainsi de l'ambiance, "atmosphère matérielle ou morale qui environne une personne » (Daro, 2011). Ces ambiances relèvent de réception de signaux émis par les formes architecturales, non seulement visuelles (lieux fermés) mais aussi vécues (Belakehal, 2012). Les propriétés morphologiques des lieux déterminent alors les attitudes à tenir dans certains services où chaque mouvement dans l'espace semble contrôlé, observé et soumis à des règles strictes, celles de l'hygiène et de la surveillance de la diffusion des germes. L'ambiance est le produit d'une interaction complexe entre : un contexte (le domaine des soins), un environnement (l'hôpital), un usager (aux usages et perceptions variés) et un espace architectural (qui se doit d'être en conformité avec l'objectif des lieux : soigner).

\section{Des seuils pour réguler les mouvements} (maternité, urgences, etc) qui constituent autant de « lieux synaptiques », remplissant un rôle de seuils accompagnés de rituels symboliques liés à la fonction du lieu, comme le trajet pour accéder à une chambre. Ces services créent des filtres destinés à limiter peu à peu les mouvements jusqu'au cœur d'un schéma où peu de personnes accèdent : les services administratifs et de recherche médicale. laisse entrevoir les limites imposées à la liberté de circulation et les contraintes liées à la fonction médicale du site.

Les seuils ne se retrouvent pas forcément là où on les attend dans les hôpitaux burkinabè. La hiérarchie établit des rapports de force qui s'inscrivent dans l'espace (Petit, 2010), concrètement matérialisés par les espaces non accessibles à l'intérieur de l'hôpital pédiatrique. Ainsi, les relations de pouvoir s'imposent aux personnes séjournant au sein de l'établissement, d'où une forme de réclusion au sein des unités de soins. Les filtres sont matérialisés par les vigiles et les murets.

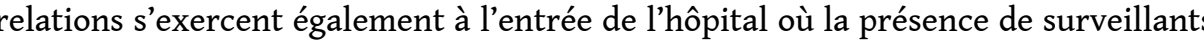
régule les entrées. Toutefois, elles ne se traduisent pas toujours de la même manière que dans les pays du Nord où « un personnel d'accueil peut être positionné de sorte à constituer un barrage entre les experts et le public " (Petit, 2010). Preuve en est, le mécontentement des médecins des urgences qui se plaignent de recevoir trop souvent des malades mal orientés.

\section{Des espaces hors du modèle de contrôle}

Entre projection identitaire (Larceneux, 2011) et liens affectifs avec les lieux (Audas, 2012), de nouvelles fonctions sont créées par appropriation de lieux dont la destination

EchoGéo, 30 | 2014 
initiale était tout autre. Ainsi, alors que la recherche d'espace privatif est une préoccupation au Nord (Curtis et al, 2007), dans les pays du Sud, les espaces de sociabilité sont recherchés, voire créés, par les familles des patients. Dans la mosaïque des lieux créés par les architectes (blocs d'hospitalisation, cour intérieure, etc.), les paillotes constituent les lieux dont la fonction est la plus fréquemment détournée : pour la pratique des rites religieux, à l'entrée de l'hôpital à la vue de tous pour les musulmans, ou plus cachés près des bâtiments maladies infectieuses pour les chrétiens. L'usage des paillotes est repensé au rythme des prières. L'appropriation spontanée des lieux est la réplique de pratiques routinières qui s'exercent dans le quotidien hors de l'espace hospitalier mais qui n'a pas été pensé lors de la conception du site. Elles permettent de rassurer les patients et leur famille, en offrant une infime reproduction de l'espace vécu (Frémont, 1981).

Enfin, à l'exception de l'unité "maladies infectieuses », et malgré l'absence de limites physiques pourtant très présentes sur le territoire hospitalier, les bâtiments de $3^{\text {ème }}$ ligne forment un espace aux marges de l'hôpital. Leur situation géographique, comme reléguée en périphérie d'un espace complètement fermé vers l'extérieur dans cette partie de l'hôpital, participe à sa distanciation. Elle est renforcée par l'existence de fonctions symboliquement négatives (incinérateur de déchets, morgue) qui confèrent une image répulsive à ces lieux.

\section{Conclusion}

En raison de l'importance des services de soins offerts, de leur spécificité et de leur capacité d'accueil, les hôpitaux sont généralement observés à travers les activités menées et leur territoire d'attraction. Leur insertion dans la ville, leur accessibilité constituent des préoccupations pour les aménageurs. Or, l'intérêt apporté par les architectes à la structure hospitalière montre que cet établissement peut être observé comme un territoire à part entière à travers son organisation.

L'hôpital n'est pas un simple rassemblement d'équipements, c'est un espace où la juxtaposition des lieux de soins, qui n'est pas neutre, est destinée à orienter le comportement des utilisateurs. Ces éléments participent au bien-être des hospitalisés et concourent également à l'amélioration des activités des soignants. Ainsi, l'organisation des flux de déplacement, la disposition des bâtiments et l'inaccessibilité de certains couloirs, montrent la volonté de maitriser les personnes entrant dans l'hôpital. La limitation des mouvements imposée par l'organisation interne de l'hôpital est reflétée par la simplicité des itinéraires de déplacement. Une attitude non conforme à ce qui est attendu peut entraîner une marginalisation. De ce fait, et malgré des efforts architecturaux pour reproduire le modèle d'habitat local, l'espace hospitalier demeure à l'écart de l'espace vécu des malades.

L'organisation des lieux fondée sur l'existence de frontières, réelles ou perçues, de seuils et d'espaces «d'exclusion » participe à un sentiment d'égarement exprimé par tous les enquêtés. La perte de repères spatiaux observée dans l'enceinte de l'hôpital pourrait être mise en parallèle avec « l'égarement médical » au sein des services. Il met en évidence le rôle primordial du paysage thérapeutique sur le bien-être des patients et la nécessaire approche infra-hospitalière pour en comprendre tous les effets. 


\section{BIBLIOGRAPHY}

Andrade C. et al, 2012. Users views of hospital environmental quality: Validation of the Perceived Hospital Environment Quality Indicators (PHEQIs). Journal of environmental psychology, 32 , p. 97-111.

Audas N., 2012. Les prises affectives des lieux en tant qu'expression de l'ambiance urbaine. Actes $d u 2^{\text {nd }}$ international congress on ambiances, Montréal 19-22 sept. 2012, p. 345-350.

Audas N., 2008. De l'espace fonctionnel à l'espace vécu : les modes d'appropriation affective d'un archétype du non-lieu, la gare. Colloque « Espaces de vie, espaces-enjeux : entre investissements ordinaires et mobilisations politiques ", Rennes 2008, 14 p.

Balique H., 2004. L'hôpital public en Afrique francophone. Médecine tropicale, 64, p. 545-511.

Belakehal A., 2012. Ambiances patrimoniales. Problèmes et méthodes. Ambiances en acte(s) International Congress on Ambiances, Montreal 2012, Canada, p. 505-510.

Borasi G., Zardini M., 2012. Pour une médicalisation de l'architecture. In En imparfaite santé, la médicalisation de l'architecture, Centre canadien d'architecture, Canada et Lars Müller publishers, Suisse, $24 \mathrm{p}$.

Briand P., Pary A., 2011. L'hôpital: un indicateur de solidarité sociale. Centrale sanitaire suisse romande, cahier $\mathrm{n}^{\circ} 5$, p. 3 .

Burton R., s.d. L'hôpital St Mary sur l'île de Wight, 2 p, http://www.culture.gouv.fr/culture/ politique-culturelle/architecture.pdf

Cantal-Dupart M., 2012. Un hôpital urbain, idéalement implanté dans la ville. Les tribunes de la santé, Presses de Sciences Po, n 37, p. 75-81.

Chenge Mukalenge F., 2013. De la nécessité d'adapter le modèle de district sanitaire au contexte urbain : exemple de la ville de Lubumbashi en RD Congo. Studies in Health Services Organisation \& Policy, 31, $131 \mathrm{p}$.

Clémençon AS, 2002. Le rôle de l'institution hospitalière dans l'urbanisation : le cas des Hospices civils de Lyon. Actes du colloque Hôpital, urbanisme et architecture, Grand Lyon Mission Prospective et Stratégie d'agglomération, p 13-14.

Curtis S., Gesler W., Fabian K., Francis S., Priebe S., 2007. Therapeutic landscapes in hospital design: a qualitative assessment by staff and service users of the design of a new mental health inpatient unit, Environment and Planning C: Government and Policy, volume 25, p 591-610.

Daro C., 2011. Ambiance et environnement : quelques repères en architecture. Actes 1st International Congress on Ambiances, Grenoble 2008, Édition À La Croisée, p. 203-207.

Déverin Y., 1999. De la concession rurale à la parcelle urbaine. Mutations de l'habitat en pays mossi (région de Ouagadougou, Burkina Faso). Les annales de la recherche urbaine, 85, p. 132-139.

Di Méo G., 2008. Espaces d'enfermement, espaces clos : l'esquisse d'une problématique. Cahiers ADES, Actes du colloque «Espace d'enfermement, espaces clos », 20 mai 2008, Bordeaux, p. 7-18.

Djouda Feudjo YB., 2010. Réseaux relationnels et processus de soutien aux malades de la tuberculose au Cameroun. REDES, $n^{\circ} 6,18 \mathrm{p}$.

Dozon J.-P., Bourdillon F., 2012. La maladie est une affaire éminemment sociale. Les tribunes de la santé, Presses de Sciences Po, $\mathrm{n}^{\circ}$ 43, p. 101-104 
Durand JP, Le Floch MC, 2006. Conceptualisation et processus de connaissance face au consentement au travail. L'Harmattan, collection Logiques sociales, Paris, 318 p.

Ebang Ondo E., 2012. Perception de l'hôpital public et offre de soins de santé au Gabon : analyse des enjeux des interactions entre personnels et usagers du Centre Hospitalier de Libreville (CHL). Bulletin Amades, $\mathrm{n}^{\circ}$ 85, 6 p.

Fermand C., 2002. Pour une histoire urbaine et architecturale de l'hôpital. Cahier Millénaire 3, Actes du colloque " Hôpital, urbanisme et architecture ", 3 avril 2002, Hôtel de la Communauté urbaine de Lyon, p. 10-11.

Fleuret S., 2003. Recomposition du paysage sanitaire : quand les exigences territoriales rejoignent les enjeux de santé. Géocarrefour, Santé et territoires, 78/3, p. 239-245.

Fleuret S., 2012. L'évolution des savoirs en santé et la place de la médecine dans la société, dans les territoires et dans la ville. Espaces et sociétés, UMR 6590 CNRS, Université d'Angers, 33, p. 43-55. Frémont A., 1981. Ouvriers et ouvrières à Caen - Les lieux de la vie. Norois, 112, p. 615-627. George P. 1966. L'Hôpital et la Cité, de R. F. Bridgeman. Annales de Géographie, t. 75, nº 407, p. 96-97.

Gesler W., 1992. Therapeutic landscapes: medical issues in light of the new cultural geography. Social science and medicine, vol 34, $\mathrm{n}^{\circ} 7, \mathrm{p}$ 735-746.

Gesler W., Belle M., Curtis S., Hubbard P., Francis S., 2004. Therapy by design: evaluating the UK hospital building program. Health and place, 10, p. 117-128.

Granier P., 2002. Les espaces de l'hôpital, analyse multiscalaire d'une organisation hospitalière, le CHRU de Tours. Thèse de Géographie, Université de Tours, 360 p.

Herranz G., Thomas H., 2009. L'hôpital en tant que sujet moral. Institut européen de bioéthique, 13 p. Jaffré Y., Olivier de Sardan JP., 2005. Une médecine inhospitalière. APAD-Karthala, Paris, 462 p.

Labasse J., 1980. L'hôpital et la ville. Géographie hospitalière. Hermann, Paris, 240 p.

Larceneux F., 2011. J’habite donc je suis. Etudes foncières, $\mathrm{n}^{\circ} 23-26,6$ p.

Luisier V., 2011, «J'ai mal à l'hôpital» : les promesses non tenues de la médecine hospitalière. Centrale sanitaire suisse romande, cahier $\mathrm{n}^{\circ} 5, \mathrm{p} .1$.

Loschak D., 2007. Espace et contrôle social, http://www.u-picardie.fr/labo/curapp/revues/root/8/ loschak.pdf

Manga Zambo E., nd. Les transformations de la gestion hospitalière dans leurs modernes expressions juridiques et administratives au Cameroun. Cahiers africains d'administration publique, $15 \mathrm{p}$.

Mas B., Pierru F., Smolski N., Torrielli R., 2011. L'hôpital en réanimation. Éditions du croquant, introduction, $17 \mathrm{p}$.

Moullé F., 2010. De la frontière aux discontinuités. Regards de géographes. Esprit d'avant, bulletin $\mathrm{n}^{\circ} 10,10 \mathrm{p}$.

Nikiema A, Rossier C, Millogo R, Ridde V, 2011. Inégalités de l'accès aux soins en milieu urbain africain : le cas de la périphérie nord de Ouagadougou. Communication à la $6^{e}$ conférence africaine sur la population « Population africaine : passé, présent et futur », UEPA, Ouagadougou, Burkina Faso, 16 p.

OMS, 1946. Préambule à la constitution de l'Organisation mondiale de la santé. Conférence internationale sur la Santé, New York, 19-22 juin 1946. 
Petit M., 2010. Le paysage thérapeutique : éléments de différenciation de l'offre de soins? Mémoire de M2 géographie, Université de Rouen, 109 p.

Roodenbeke E. de, 2003. Privatisation des hôpitaux dans les pays en voie de développement ? 15 p, info.worldbank.org/etools/docs/library/232765/Reform Hospitaliere/docs/CDR/ privathopitauxPED.pdf

Shadyc-Gril, 2002. Une anthropologie de la fange. PDM, PSeau, 223 p.

UNAF, 2007. Les chartes dans le domaine de la santé. Paris, 82 p.

Vaguet A., Lefebvre B., Petit M., 2012. Lieux de soins et paysages thérapeutiques. Du concept à la méthode. BAFG Géographies, ${ }^{\circ} 2$, $\mathrm{p}$ 214-223.

\section{ABSTRACTS}

In geography, hospital is rarely observed at small scale. Surveys in children's hospital of Charles de Gaulle were the occasion to approach the places which compose the hospital. The organization of the establishment is described and axes of movement were observed. The objective is to include elements which compose the therapeutic landscape and to define a model of organization.

En géographie, l'hôpital est rarement observé à une échelle infrahospitalière. Les enquêtes menées à l'hôpital pédiatrique Charles de Gaulle de Ouagadougou ont été l'occasion d'approcher les lieux qui composent l'hôpital. L'organisation de cet établissement est décrite et les axes de déplacement observés. L'objectif est de comprendre les éléments qui composent le paysage thérapeutique et d'esquisser un modèle d'organisation.

\section{INDEX}

Keywords: hospital, urban, movement, health geography, Ouagadougou

Mots-clés: espace hospitalier, ville, déplacement, géographie de la santé, Ouagadougou

\section{AUTHOR}

\section{AUDE NIKIEMA}

Aude Nikiema, nikiaude@yahoo.fr, est Chargée de recherches à l'INstitut des Sciences des Sociétés (INSS), Ouagadougou. 